\title{
Silicon Photonics for Computing Systems
}

\author{
JIANG XU, Hong Kong University of Science and Technology \\ YUICHI NAKAMURA, System Platform Research Labs, NEC \\ ANDREW KAHNG, University of California, San Diego
}

Computing systems, from HPC and data center to automobile, aircraft, and cellphone, are integrating growing numbers of processors, accelerators, memories, and peripherals to meet the burgeoning performance requirements of new applications under tight cost, energy, thermal, space, and weight constraints. Recent advances in photonics technologies promise ultra-high bandwidth, low latency, and great energy efficiency to alleviate the inter-/intra-rack, inter-/intra-board, and inter-/intra-chip communication bottlenecks in computing systems. Silicon photonics technologies piggyback onto developed silicon fabrication processes to provide viable and cost-effective solutions. Many companies and institutes have been actively developing silicon photonics technologies for more than a decade. Many silicon photonics devices and circuits have been demonstrated in CMOS-compatible fabrication processes. Silicon photonics technologies open new opportunities for applications, architectures, design techniques, and design automation tools to fully explore new approaches and address the challenges of next-generation computing systems.

With strong interests from both industry and academia, two international workshops, PHOTONICS (photonics-optics technology oriented networking, information and computing systems) and OPTICS (optical/photonic interconnects for computing systems), have been held annually since 2015. The Special Issue on Silicon Photonics presents the latest progress and provides insights into the challenges and future developments of this emerging area. Several works presented during the workshops are expanded and included in this special issue.

This special issue received 18 submissions, and we assigned each manuscript to at least three reviewers. After two rounds of reviews in a similar process as regular articles, seven works were selected for the special issue. The works presented in this special issue span through challenges, design techniques, key building blocks, and architectures of silicon photonics for computing systems.

The first work is "A Learning-Based Thermal-Sensitive Power Optimization Approach for Optical NoC" by Zhang and Ye. It targets thermal challenges of silicon photonics, and the authors propose a learning-based thermal-sensitive power optimization approach for optical networks-on-chip under temperature variations. In "A Process Variation Tolerant Method for Nanophotonic On-Chip Network," Xu et al. target silicon photonic process variations and propose a series of solutions to the wavelength drifting issue of microring resonators and subsequent bandwidth loss of optical networks due to process variation.

Two works on the energy efficiency of silicon photonics technologies are included in this issue. In "Reducing Power Consumption of Lasers in Photonic NoCs Through Application-Specific Mapping," Fusella and Cilardo propose a design space exploration methodology for optical NoC mapping to improve energy efficiency. Luo et al. propose an off-line approach to co-optimize laser power levels and application execution times in their work, titled "Offline Optimization of Wavelength Allocation and Laser Power in Nanophotonic Interconnects.”

2018 Copyright is held by the owner/author(s).

1550-4832/2018/07-ART20

https://doi.org/10.1145/3208198 
Key silicon photonic building blocks and architectures are presented in three works. Ishihara et al. introduce optical pass-gate logic and propose a parallel adder based on the optical passgate logic in their work, titled "An Integrated Nanophotonic Parallel Adder." VanWinkle and Kodi propose an optical network-on-chip for CPU-GPU systems in "SHARP: Shared Heterogeneous Architecture with Reconfigurable Photonic Network-on-Chip.” In "Integrated High Speed Optical SerDes over 100GBd Based on Optical Time Division Multiplexing," Xu et al. propose a novel integrated optical transceiver for silicon photonic systems.

The guest editors acknowledge the Editor-in-Chief, Dr. Yuan Xie, for his vision and support on this special issue. We are very thankful to all the reviewers for their time and effort in sharing their expertise to improve each work. The guest editors would like to congratulate the authors for their excellent work and contributions. We sincerely hope that this special issue will help to actively explore silicon photonics technologies for computing systems. 